\title{
Charge trapping and detrapping in polymeric materials: Trapping parameters
}

\author{
Tian-chun Zhou, ${ }^{1,2, a)}$ George Chen, ${ }^{2}$ Rui-jin Liao, ${ }^{1}$ and Zhiqiang $\mathrm{Xu}^{2}$ \\ ${ }^{1}$ State Key Laboratory of Power Transmission Equipment \& System Security and New Technology, \\ Chongqing University, Chongqing, China, 400044 \\ ${ }^{2}$ School of Electronics and Computer Science, University of Southampton, Southampton, Hampshire, \\ United Kingdom, SO17 1BJ
}

(Received 17 January 2011; accepted 18 July 2011; published online 31 August 2011)

\begin{abstract}
Space charge formation in polymeric materials can cause some serious concern for design engineers as the electric field may severely be distorted, leading to part of the material being overstressed. This may result in material degradation and possibly premature failure at the worst. It is therefore important to understand charge generation, trapping, and detrapping processes in the material. Trap depths and density of trapping states in materials are important as they are potentially related to microstructure of the material. Changes in these parameters may reflect the aging taken place in the material. In the present paper, characteristics of charge trapping and detrapping in low density polyethylene (LDPE) under dc electric field have been investigated using the pulsed electroacoustic (PEA) technique. A simple trapping and detrapping model based on two trapping levels has been used to qualitatively explain the observation. Numerical simulation based on the above model has been carried out to extract parameters related to trapping characteristics in the material. It has been found that the space charge decaying during the first few hundred seconds corresponding to the fast changing part of the slope was trapped with the shallow trap depth 0.88 $\mathrm{eV}$, with trap density $1.47 \times 10^{20} \mathrm{~m}^{-3}$ in the sample volume measured. At the same time, the space charge that decays at longer time corresponding to the slower part of the slope was trapped with the deep trap depth $1.01 \mathrm{eV}$, with its trap density $3.54 \times 10^{18} \mathrm{~m}^{-3}$. The results also indicate that trap depths and density of both shallow and deep traps may be used as aging markers as changes in the material will certainly affect trapping characteristics in terms of trap depth and density. (C) 2011 American Institute of Physics. [doi:10.1063/1.3626468]
\end{abstract}

\section{INTRODUCTION}

Space charge formation in polymeric materials can cause some serious concern for design engineers as the electric field may severely be distorted, leading to part of the material being overstressed. At the worst, this may result in material degradation and possibly premature failure. ${ }^{1-3}$

A thorough understanding of the dielectric properties of polymeric insulating materials is important to efficiently and safely utilize the materials in both power and electronic industries. Recently, the phenomenon of charge trapping and detrapping has attracted significant attention, since charge characteristics of the insulating material may be closely related to the aging taken place in it. ${ }^{4,5}$

However, to determinate the trapping parameters and characteristics of the material is not an easy task and various methods have been developed to understand charge dynamics when subjected to electric fields. Thermally stimulated currents (TSC) technology has been considered as one of the efficient measuring methods to determinate the trapping parameters, ${ }^{6}$ but it is a destructive method as it generally alters morphologic features of the material due to heating. The development in space charge mapping techniques ${ }^{7}$ in the past few decades has resulted in an abundant knowledge resource on space charge, and one of the most popular methods is the pulsed electroacoustic (PEA) measurement. One of the

\footnotetext{
a) Author to whom correspondence should be addressed. Electronic mail: ztccqu@163.com.
}

advantages of the PEA method is a non-destructive measurement which is safe for the insulation of the material, so it is more convenient and possible for the condition monitoring of the insulating states of power equipments. There are a large number of papers about the PEA measurement and its space-charge profile description, but space-charge accumulation and the related trapping-detrapping phenomena are still poorly understood. ${ }^{8}$ One of the techniques to characterize traps is to investigate the time characteristics of charge trapping and detrapping.

In our previous paper, ${ }^{9}$ a theoretical model based on shallow and deep traps has been proposed to describe space charge dynamics considering both trapping and detrapping process. In this paper, a quantitative analysis of the trapping parameters and characteristics has been carried out using our experimental data via PEA measurement on low density polyethylene (LDPE) samples. The results indicate that trap depths and density of both shallow and deep traps may be used as ageing markers as changes in the material will certainly affect trapping characteristics in terms of trap depth and density.

\section{BRIEF REVIEW OF THEORETICAL MODEL OF TWO LEVEL TRAPS}

Trapping and detrapping are closely related to the trap distribution in terms of spatial and energy depths. From aspect of energy, for a semicrystalline polymeric material, it is possible that traps may have several discrete energy depths 
or even continuous energy distribution. Spatially, trap distribution may vary for a sample as the material may experience different processes including physical, thermal, and chemical treatment. This is especially true for the region near to the surface. To simplify the mathematics involved here we assume that the traps are uniformly distributed across the sample and have only two trap energy levels, i.e., one representing shallow trap and another for deep trap.

The trapping model can be described by three processes as following: charge injection, trapping, detrapping and kinetics of trapping and detrapping process, respectively. To simplify the model, charge injection process is assumed to obey Schottky injection and tunneling, and generally, the current increases exponentially with the applied field and decays exponentially with the time. The trapping process from shallow to deep trap and the detrapping process from deep trap to shallow trap are not considered in the present study. In addition, we assume the trapped charges are close to the injecting electrode, so the recombination with the injected charge from the opposite electrode can be neglected. The details of the model description can be found in Ref. 9.

Based on the above assumption, the kinetics of trapping and detrapping process can be expressed as follows:

$$
\begin{aligned}
& \frac{d n_{1}}{d t}=\frac{J \sigma_{1}}{q}\left(N_{1}-n_{1}\right)-k_{t h 1} n_{1}, \\
& \frac{d n_{2}}{d t}=\frac{J \sigma_{2}}{q}\left(N_{2}-n_{2}\right)-k_{t h 2} n_{2} .
\end{aligned}
$$

Assume the injected current depends on the applied field only, i.e., the equation of $J(\mathrm{E})$ becomes $J(\mathrm{E})=J_{0} \exp \left(E / E_{0}\right)$.

Where $N_{1}$ and $N_{2}$ are the total number density of shallow and deep traps, $\sigma_{1}, \sigma_{2}$ are trapping cross section for shallow and deep trap, and $n_{1}$ and $n_{2}$ represent the number density of trapped charges in shallow and deep trap, respectively. $q$ is the charge amount of the electron, $J_{0}$ is the initial current density when the applied field is $E_{0}, E$ is the applied field. $k_{\mathrm{th} 1}$ and $k_{\mathrm{th} 2}$ are the thermal detrapping rate constants for shallow and deep traps respectively. The thermal detrapping rate constant can be express as

$$
k_{t h}=N_{c} v_{t h} \sigma_{c} \exp \left[-\frac{E_{t}}{k T}\right] .
$$

The first term on the right hand side of the Eqs. (1) and (2) represents the rate change of the trapping process, and the second term the rate of change of the detrapping process. In this paper, the emphasis is placed on charge decay after removing the applied field. The detrapping process after the removal of the applied field is as follows.

$$
\begin{aligned}
& n_{1}(t)=n_{10} \exp \left(-k_{t h 1} t\right), \\
& n_{2}(t)=n_{20} \exp \left(-k_{t h 2} t\right),
\end{aligned}
$$

where $N_{\mathrm{c}}$ is the effective density of states in the conduction band, $v_{\text {th }}$ is the thermal velocity of the charge, $E_{\mathrm{t}}$ is the trap depth, $k$ is the Boltzmann constant, and $T$ is the temperature $n_{10}$ and $n_{20}$ are the initial number density of trapped charges in shallow and deep trap at the moment when the applied field is removed, respectively.

The number density of total trapped charge density during detrapping process is given by

$$
n(t)=n_{1}(t)+n_{2}(t)=n_{10} \exp \left(-k_{t h 1} t\right)+n_{20} \exp \left(-k_{t h 2} t\right) .
$$

The parameters $k_{\mathrm{th} 1}, \mathrm{k}_{\mathrm{th} 2}, n_{10}$, and $n_{20}$ are important as they are potentially related to microstructure of the material. Changes in parameters may reflect ageing taken place in the material. In details, $k_{\mathrm{th} 1}$ and $k_{\mathrm{th} 2}$ reflect two different trap depths in the material which may be related to different kinds of chemical or physical defects in the material. Likeness, $n_{10}$ and $n_{20}$ may be related to the trap density of both shallow and deep traps which will be further discussed in the next section. The above four parameters can be obtained via the data from a series of space charge decay measurements for different stressing times.

\section{SPACE CHARGE DECAY EXPERIMENTAL RESULTS FROM LPDE}

Space charge measurements were carried out on additive-free LDPE using the PEA technique. The thickness of the film was $\sim 180 \mu \mathrm{m}$. Details of the experimental can be found in Ref. 10. Figure 1 shows the changes in space charge profiles during decay process after the sample being stressed at $4 \mathrm{kV}$ for 10 mins. Because of attenuation and dispersion of the acoustic wave during transit of the sample the estimated magnitude of the space charge near the anode electrode is less accurate than that closer to the piezoelectric detector located beneath the cathode electrode. For this reason, the analysis of the space charge decay will be concentrated on the space charge close to the cathode and the kind of the charge carrier is the electron. The total amount of space charge in the bulk and the number density of the total trapped charge can be estimated using the following equation, respectively,

$$
\begin{gathered}
Q=\int_{0}^{d}|\rho(x)| \bullet S \bullet d x, \\
n=Q /(S \bullet d \bullet q),
\end{gathered}
$$

where $\rho(x)$ is charge density, $S$ is the electrode area and $d$ is the thickness of the layer close to the cathode. The value of $\rho(x)$ is shown in Fig. 1, and $S$ and $d$ used in the experiments are $63.585 \mathrm{~mm}^{2}$ (radius of the electrode area is $4.5 \mathrm{~mm}$ ) and $\sim 50 \mu \mathrm{m}$, respectively. A similar measurement has been performed on the sample that has been stressed at the same voltage but for a short time (2 mins). The influence of stressing duration on trapping and detrapping characteristics will be discussed in the next section. According to Eq. (7), the total trapped charge can be calculated and plotted in Fig. 2. It has been found that the total trapped charge $Q(\mathrm{t})$ decreases fast at first and is followed by a slow decay. Such behaviors indicate that there are two kinds of traps existing in the material as described in Sec. II. 


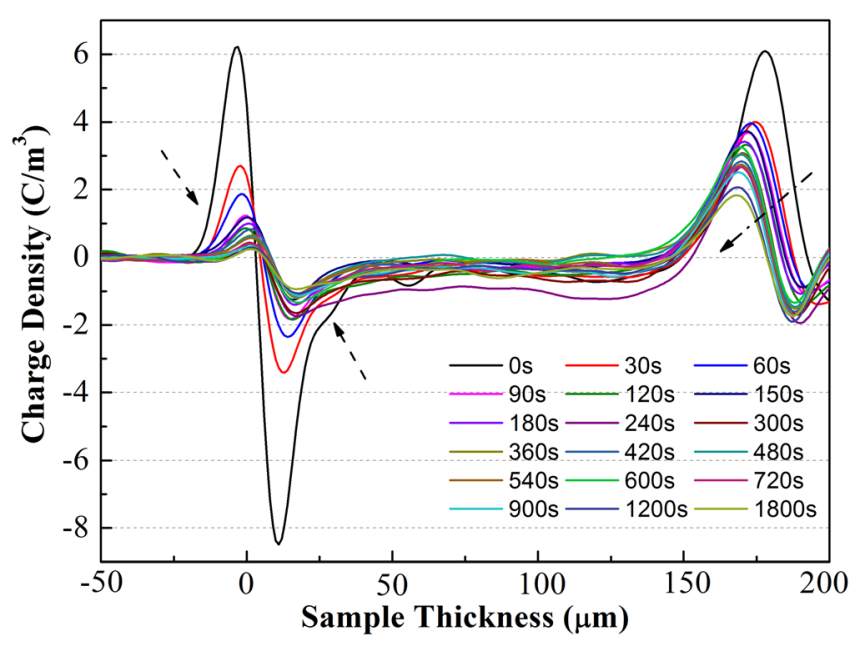

FIG. 1. (Color online) Space charge decay after the removal of the applied voltage $4 \mathrm{kV}$ for $\mathrm{t}=10 \mathrm{~min}$.

\section{CALCULATION OF TRAP PARAMETERS}

\section{A. Calculation of parameters in the model}

Numerical simulation is carried out to extract parameters from the model. The experimental data in Sec. III can be fitted well considering Eqs. between (6) and (8). The Least Squares Nonlinear Regression Analysis is used to fit the data, and the type of fit is chosen as the dual-exponential by using the Trust-Region algorithm. All these process can be easily carried out by a simple MATLAB program or some other fitting tools. Fitting results of the calculation of the parameters $k_{\text {th } 1}, k_{\text {th } 2}$ and $n_{10}$, and $n_{20}$ are shown in Table I and Fig. 3.

It has been found that for 2 mins and 10 mins stressing, both of shallow and deep traps in the material can simultaneously capture charges. However, the number density of trapped charges in shallow traps $\left(n_{10}\right)$ is 16 times more than that in deep traps $\left(n_{20}\right)$. This demonstrates that charges are easier trapped in shallow traps than deep traps. It can also be shown that the thermal detrapping rate constants $k_{\mathrm{th} 1}$ and $k_{\mathrm{th} 2}$ have a significant difference numerically. $k_{\mathrm{th} 1}$, which reflects the changing rate of shallow traps, are about 100 times more than $k_{\mathrm{th} 2}$, which stands for the changing rate of deep traps. This means that charges decay faster from shallow traps than that from deep traps.

Comparing 2 mins and 10 mins stressing, the number density of both shallow and deep trapped charges 10 mins stressing is just a little more than that at 2 mins stressing.

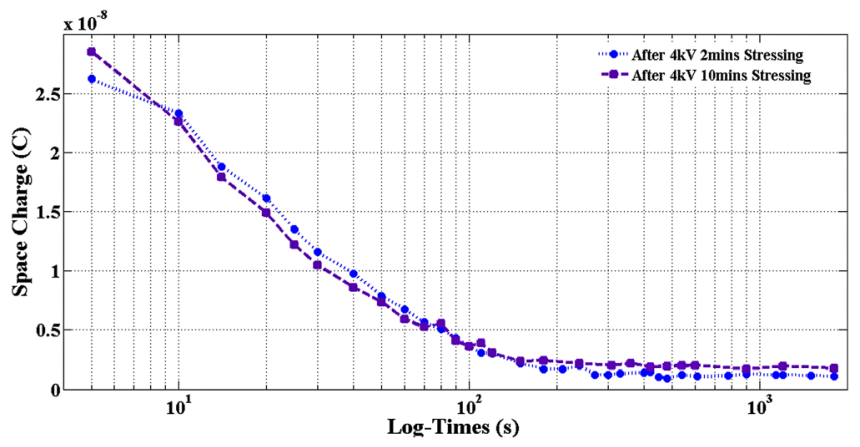

FIG. 2. (Color online) Decay of the total trapped charge after being stressed at $4 \mathrm{kV}$ for $\mathrm{t}=2$ and $10 \mathrm{~min}$, respectively.
TABLE I. Parameters derived from fitting data for the shallow and deep trap model.

\begin{tabular}{lcc}
\hline \hline Parameters & $\begin{array}{c}2 \text { mins of stressing } \\
\left(R^{2}=99 \%\right)\end{array}$ & $\begin{array}{c}10 \text { mins of stressing } \\
\left(R^{2}=99 \%\right)\end{array}$ \\
\hline$Q_{10}(\mathrm{C})$ & $2.79 \times 10^{-8}$ & $2.80 \times 10^{-8}$ \\
$Q_{20}(\mathrm{C})$ & $1.72 \times 10^{-9}$ & $1.78 \times 10^{-9}$ \\
$n_{10}=Q_{10} /(S d q)\left(\mathrm{m}^{-3}\right)$ & $5.48 \times 10^{19}$ & $5.50 \times 10^{19}$ \\
$n_{20}=Q_{10} /(S d q)\left(\mathrm{m}^{-3}\right)$ & $3.38 \times 10^{18}$ & $3.49 \times 10^{18}$ \\
$k_{\mathrm{th} 1}\left(\mathrm{~s}^{-1}\right)$ & & 0.02996 \\
$k_{\mathrm{th} 2}\left(\mathrm{~s}^{-1}\right)$ & & 0.000373 \\
\hline \hline
\end{tabular}

This is because the total charges calculated are close to the electrode and the most traps are filled with the injected charges within 2 mins. The thermal detrapping rate constants should not alter with stressing duration unless there is change in trapping depth in the material. Our calculation results are agreeable to this. The values for both stressing time are the same values, therefore, the ratio of $k_{\mathrm{th} 1}$ and $k_{\mathrm{th} 2}$ at two different stressing times is also the same.

\section{B. Trap density}

At the same time, results of the trap density of shallow and deep traps can be obtained from our experimental data, as can been seen that charges in both shallow and deep traps increase with the time duration from our experimental analysis. The order of magnitude of the density of trapped charges in our calculation is agreeable to the results obtained using electroluminescence (EL) emission detection of the LDPE material. $^{11}$

According to our proposed new model, we can present the expression of the $n_{10}$ and $n_{20}$, as expressed in Eqs. (9) and (10) from integration of Eqs. (1) and (2) as follows:

$$
\begin{aligned}
& n_{10}=\frac{A_{1} N_{1}}{A_{1}+k_{t h 1}}\left\{1-\exp \left[-\left(A_{1}+k_{t h 1}\right)\right] t\right\}, \\
& n_{20}=\frac{A_{2} N_{2}}{A_{2}+k_{t h 2}}\left\{1-\exp \left[-\left(A_{2}+k_{t h 2}\right)\right] t\right\} .
\end{aligned}
$$

The shallow trapped charges are also relevant to parameters $A_{1}, k_{\text {th } 1}$, and $N_{1}$, and the deep trapped charges are related to $A_{2}, k_{\text {th2 }}$ and $N_{2}$. It is possible to compare the amount of $N_{1}$ and $N_{2}$ if knowing the above all parameters $\left(A_{1}, A_{2}, k_{\mathrm{th} 1}\right.$ and $\left.k_{\mathrm{th} 2}\right)$. The thermal detrapping rate constants for shallow and deep traps $k_{\text {th1 }}$ and $k_{\text {th2 }}$ can be obtained from the space charge decay results directly. Now what is left is the determination of $A_{1}$ and $A_{2}$. Given the data shown in Table $\mathrm{I}$ on the value of $n_{10}$ for two different poling times $120 \mathrm{~s}$ and $600 \mathrm{~s}$, it is easy to estimate values for $\mathrm{A}_{1}$ and $\mathrm{A}_{2}$, and therefore, the $\mathrm{N}_{1}$ and $\mathrm{N}_{2}$ can also be estimated.

Substituting the value of $A_{1}, k_{\mathrm{th} 1}$, and $n_{10}$ and $A_{2}, k_{\mathrm{th} 2}$, and $n_{20}$ into Eqs. (9) and (10), the trap density both for the shallow $\left(N_{1}\right)$ and deep $\left(N_{2}\right)$ traps can be calculated, i.e., $N_{1}=1.47 \times 10^{20} \mathrm{~m}^{-3}$ and $N_{2}=3.54 \times 10^{18} \mathrm{~m}^{-3}$ (Shown in Table II). Comparing the value of trap density $\left(N_{1}, N_{2}\right)$ with its corresponding trapped charge density $\left(n_{10}, n_{20}\right)$ shown in Table I, it has been found that the trap density is proportional 

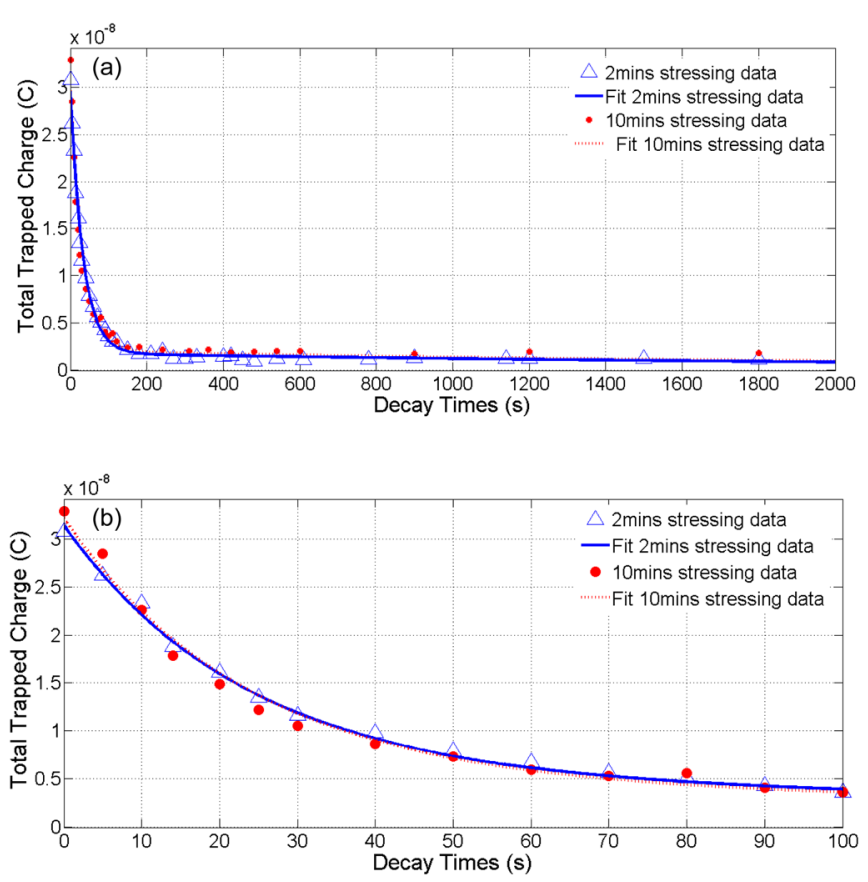

FIG. 3. (Color online) Space charge decay data and fitting for 2 and $10 \mathrm{~min}$ stressing, calculated using Eqs. (6)-(8): (a) whole view (decay times up to $1800 \mathrm{~s}$ ); (b) partial view for the first fast decay (decay times between 0 and $100 \mathrm{~s})$.

to its number density of trapped charge, then we can say the number density of trapped charge can reflect its corresponding trap density.

Obviously, the number density of shallow traps is much more than that of deep ones for our additive-free LDPE samples. This is in agreement with the conclusion that shallow traps are related to physical defects while deep traps are related to chemical defects. Since the samples used in our experiments are all unaged LDPE, indicating that physical defects should dominate charge dynamics. These findings are agreeable to the other studies where chemical impurities are most likely to be present in HDPE, or XLPE, than in LDPE, meaning that there are more deep trapping sites in HDPE and XLPE than LDPE (Ref. 12).

As can be seen that the number density of trapped charges reflect the number density of both shallow and deep traps, the space charge measurement can be an effective and sensitive method to investigate the trapping characteristics. Different materials and different situation of materials under special conditions may show different trapping characteristics, which may not be seen from some regular chemical

TABLE II. Estimation of trap density and trap depth from Eqs. (9)-(12).

\begin{tabular}{lcc}
\hline \hline Parameters & 2 mins of stressing & 10 mins of stressing \\
\hline$N_{1}\left(\mathrm{~m}^{-3}\right)$ & $1.47 \times 10^{20}$ \\
$N_{2}\left(\mathrm{~m}^{-3}\right)$ & $3.54 \times 10^{18}$ \\
$N=N_{1}+N_{2}\left(\mathrm{~m}^{-3}\right)$ & $1.51 \times 10^{20}$ \\
$A_{1}\left(\mathrm{~s}^{-1}\right)$ & 0.01784 \\
$A_{2}\left(\mathrm{~s}^{-1}\right)$ & 0.028327 \\
$E_{\mathrm{t} 1}(\mathrm{eV})$ & 0.8857 \\
$E_{\mathrm{t} 2}(\mathrm{eV})$ & 1.0117 \\
\hline \hline
\end{tabular}

method such as infrared or Raman spectra but can be sensitive to the measurement of space charge. ${ }^{13}$

\section{Trap depths}

Both physical and chemical defects may be present in insulating materials and both trap electrons. It is easy to understand that different kinds of defects may correspond to traps with different energies. To understand the link between material microstructure/composition and charge trapping characteristics, it is necessary to acquire the trap depths of shallow and deep traps in the material presented in the model.

According to Eq. (3), the trap depth can be written as:

$$
E_{t}=k T \ln \left[\frac{N_{c} v_{t h} \sigma_{c}}{k_{t h}}\right] .
$$

If all parameters in Eq. (11) are known, then the trap depth of both shallow and deep traps can be calculated. Firstly, $T$ and $k_{\text {th }}$ can be determined according to our experimental data, $k$ is the Boltzmann constant, $N_{\mathrm{c}}$ and $v_{\text {th }}$ are also constants for a specific material, $N_{\mathrm{c}}=2\left(2 \pi m_{\mathrm{e}} * k T / h^{2}\right)^{3 / 2}\left(m_{\mathrm{e}} *\right.$ is electron effective mass, $\left.m_{\mathrm{e}}{ }^{*} \approx m_{\mathrm{e}}\right)$ and its value is around the order of $10^{23} \sim 10^{25} \mathrm{~m}^{-3}$ (Ref. 14), and $v_{\text {th }}=\left(3 k T / m_{\mathrm{e}} *\right)^{1 / 2}$ and its value is in the order of $10^{5} \mathrm{~m} / \mathrm{s}$ (Ref. 15). Depending on the type of defect, a bulk trap may capture an electron or a hole that has been injected into the material. Trapping centers are generally characterized by their ability to capture cross section, $\sigma_{\mathrm{c}}\left(\mathrm{m}^{2}\right)$. Cross sections can be different based on the initial charge state of the centers, such as coulombic attractive, neutral or coulombic repulsive. For electrons the coulombic attractive center would be positively charged ranging from $10^{-16}$ to $10^{-18} \mathrm{~m}^{2}$ (Refs. 6 and 16). In the model described above, there are two kinds of traps present in the material, indicating the existence of two capture cross sections. Therefore, the values of the cross section $\sigma_{1}$ and $\sigma_{2}$ can be estimated considering the definition of $A_{1}$ and $A_{2}$ in our proposed model, ${ }^{9}$

$$
A=\frac{J_{0} \sigma_{c}}{q} \exp \left[\frac{E}{E_{0}}\right] .
$$

$J_{0}, E$, and $E_{0}$ can be obtained from the electrical conduction measurement, here we use $J_{0}=16 \mathrm{pA} / \mathrm{mm}^{2}, E=E_{0}=20$ $\mathrm{MV} / \mathrm{m}$ from our previous test results. ${ }^{17}$ Based on the above experimental data for $A_{1}$ and $A_{2}, \sigma_{1}$ and $\sigma_{2}$ can be estimated about $6.56 \times 10^{-17} \mathrm{~m}^{2}$ and $1.04 \times 10^{-16} \mathrm{~m}^{2}$, respectively. The cross section of shallow trap is smaller than that of deep one from the above calculation. Using the fitting data for $\mathrm{k}_{\mathrm{th} 1}$ and $\mathrm{k}_{\mathrm{th} 2}$ in Table $\mathrm{I}$, the shallow and deep trap depth can be estimated. The trap depth estimated by us is as follows: $E_{\mathrm{t} 1}$ is of the value $0.88 \mathrm{eV}$, and $E_{\mathrm{t} 2} 1.01 \mathrm{eV}$.

Generally, traps in the polyethylene having the trap depth energy lager than $1 \mathrm{eV}$ can be acted as deep ones, ${ }^{15,18}$ so $E_{\mathrm{t} 1}$ and $E_{\mathrm{t} 2}$ can be represented as shallow and deep trap depth energy, respectively. It must be noted here that the time delay in the PEA measurement immediately after the applied voltage was removed does not allow us to detect shallower traps. ${ }^{5}$ Our trap depth data obtained is in accordance with the value obtained by the others, ${ }^{5,19}$ and it does 
show the trapping characteristics can be described by two types of traps in the material. This supports that there may be different defects which have their own trap depth.

\section{Relationship between trap depth and its trap density}

Figure 4 shows the relationship between the trap depth and its trap density, which indicates that the space charge decaying during the first hundred seconds corresponding to the fast changing part of the slope was trapped with the shallow trap depth $0.88 \mathrm{eV}$, with a trap density of $1.47 \times 10^{20}$ $\mathrm{m}^{-3}$ in the sample volume measured. At the same time, the space charge that decays at longer time corresponding to the slower part of the slope was trapped with the deep trap depth $1.01 \mathrm{eV}$, with a trap density of $3.54 \times 10^{18} \mathrm{~m}^{-3}$. It is worth to mention that there is a gap in trap depth between the two trap ranges. This does not mean that there are no trap states in this trap depth region but just implies that any other trap states that exist only have a small trap density compared with the two main ranges for both shallow and deep trap depth characteristics values.

From Fig. 4, we can evaluate the trap characteristics of the material from both these two kinds of trap depth and its trap density. For different kinds of materials or for the same material after undergoing different conditions (for example, aging), ranges of both shallow and deep trap depth will change, also changing with their corresponding trap density.

\section{DISCUSSION}

The presence of both physical and chemical defects will lead to traps in the material having a range of trap depths. Due to aging or the presence of additives, it may lead to the accumulation of electrons in traps forming a relatively immobile space charge. ${ }^{20}$

Physical defects may be created by changing of the morphological of crystallinity ratio, molecular weight, and microstructure. All these changes may lead to a trap energy modification and trapping or detrapping process. $^{15,18,20}$ Chemical defects may be created by photo-oxidation process which will change the chemical structure of the material.

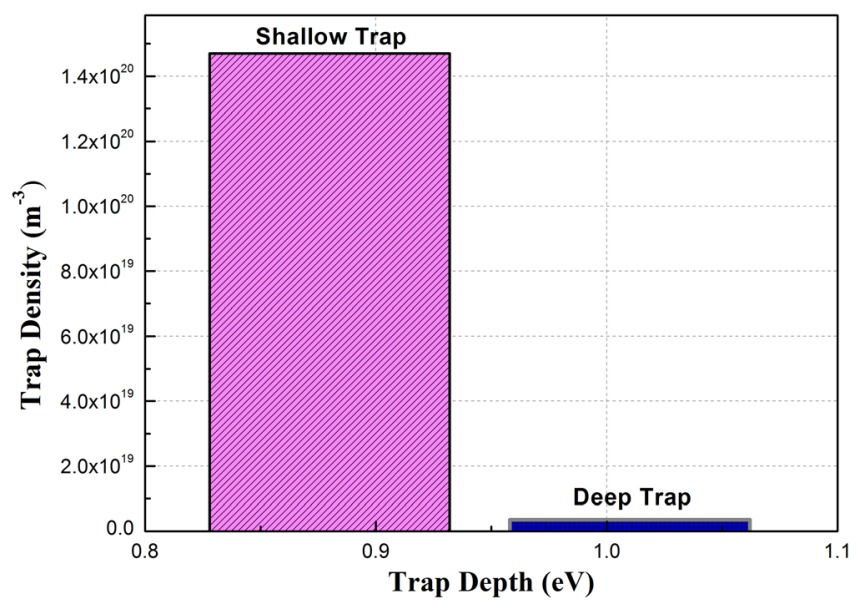

FIG. 4. (Color online) Trap depth and its trap density for shallow and deep traps.

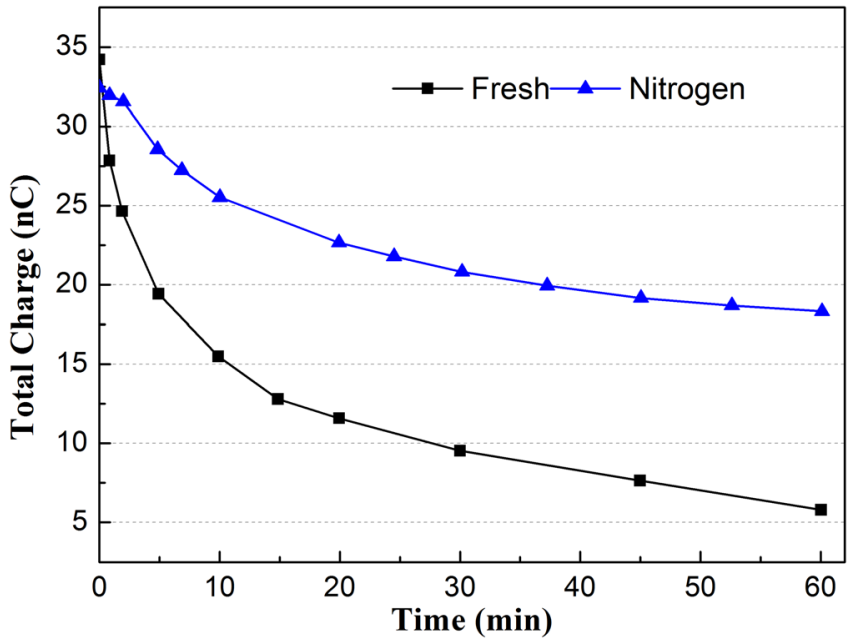

FIG. 5. (Color online) Charge decay for fresh LDPE and samples gamma irradiated in nitrogen to $100 \mathrm{kGy}$ [22].

These changes also have some influences on the trap energy distribution and trapping or detrapping process. Overall, shallow traps will have something to do with the physical defects while deep traps will be related to the chemical defects in the material. In the experimental presented in this paper, physical defects affect charging behavior stronger than the chemical defects as the material used in our study is additive-free LDPE. This is similar to the earlier research results using space charge measurement to relate the trapping and detrapping properties of polymers to their microstructures. ${ }^{21}$

To illuminate how trap characteristics reflect the state of the material, we compare the space charge decay data of both fresh and gamma irradiated in nitrogen $\left(\mathrm{N}_{2}\right)$ LDPE samples after following the application of an electric field of 50 $\mathrm{kV} / \mathrm{mm}$ for 60 mins (Ref. 22). The space charge decay of the fresh and irradiated sample is shown in Fig. 5.

From Fig. 5. it can be seen that the sample gamma irradiated in nitrogen shows the overall slower decay rate than that of fresh one. This means there are more deep traps generated after irradiated. Using our proposed model to fit the data of the samples after irradiated in nitrogen, we can get the shallow trap depth increased to $0.96 \mathrm{eV}$, with the

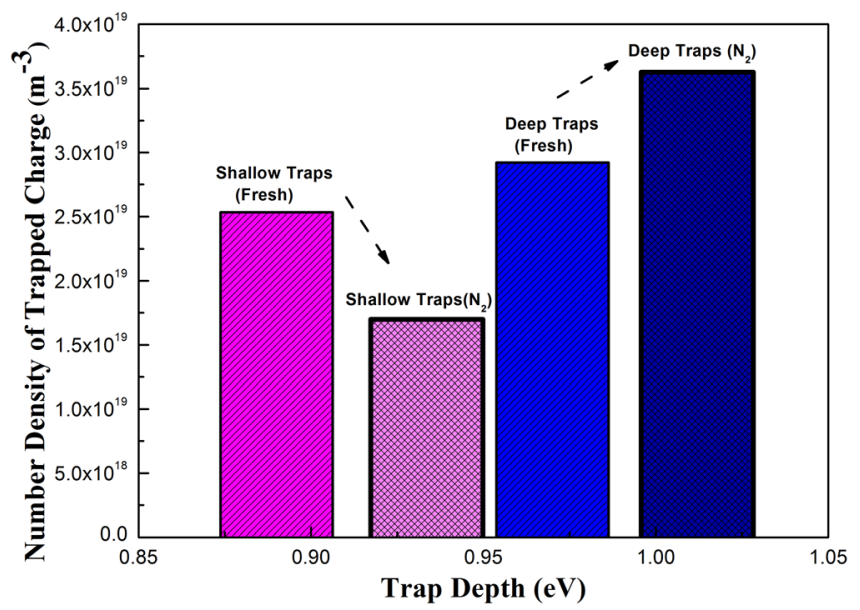

FIG. 6. (Color online) Trap depth distribution of space charge for fresh and nitrogen irradiated LDPE sample. 
corresponding number density of trapped charge $1.699 \times 10^{19} \mathrm{~m}^{-3}$, and the deeper trap depth ranges increased to $1.02 \mathrm{eV}$, with the corresponding number density of trapped charge $3.63 \times 10^{19} \mathrm{~m}^{-3}$. The result is shown in Fig. 6 , and the $Y$-axis represents the number density of trapped charge, which is proportional to the trap density shown in Fig. 4 from Eqs. (9) and (10) after a long time poling. As it can be seen that both the shallow and deep trap depth increase, and the corresponding number density of shallow trapped charge decreases while the number density of deeper trapped charge shifts to a larger amount, which implies the irradiation introduces deeper traps because the total number density of both fresh and nitrogen irradiated samples are almost the same value, that's to say there are some shallow traps transiting to the deeper traps. Due to the length of this article, the details for how the different environments influences the trap parameters will be discussed elsewhere.

\section{CONCLUSION}

From the decay of space charge of LDPE samples of different stressing time and our previous proposed new trapping model, the parameters of trapping characteristics are calculated and estimated including trap energy levels (trap depths) and number of traps (trap density) of both shallow and deep traps. It has been found that the space charge decaying during the first few hundred seconds corresponding to the fast changing part of the slope was trapped with the shallow trap depth $0.88 \mathrm{eV}$, with trap density $1.47 \times 10^{20} \mathrm{~m}^{-3}$ in the sample volume measured. At the same time, the space charge that decays at longer time corresponding to the slower part of the slope was trapped with the deep trap depth $1.01 \mathrm{eV}$, with its trap density $3.54 \times 10^{18} \mathrm{~m}^{-3}$. Finally, aging will introduce more deeper traps in the material, which indicates that trap depths and charge density of both shallow and deep traps may be used as aging marker as changes in the material.

\section{ACKNOWLEDGMENTS}

T.Z. wishes to thank the "Chinese Scholarship Council Funding for Joint Training Ph.D. Student, for the financial support, and the authors also wish to thank the financial support of
National Basic Research Program (973 Program) (Grant No. 2009CB724505-1), China and the Scientific Research Found of SKL of Power Transmission Equipment \& System Security and New Technology (Grant No. 2007DA10512708103), Chongqing University, China.

${ }^{1}$ R. Bartnilas, IEEE Trans. Dielectr. Electr. Insul. 4, 544 (1997).

${ }^{2}$ G. Blaise, J. Appl. Phys. 77, 2916 (1995).

${ }^{3}$ N. Shimuzu, Ph.D. dissertation, Nagoya University, Japan, 1979.

${ }^{4} \mathrm{G}$. Chen and T. Van Nguyen, in 2008 International Conference on Condition Monitoring and Diagnosis, 21-24 April 2008, Beijing, China (IEEE, Piscataway, NJ, 2009), pp. 633-637.

${ }^{5}$ G. Mazzanti, G. C. Montanari, and J. M. Alison, IEEE Trans. Dielectr. Electr. Insul. 10, 187 (2003).

${ }^{6}$ T. Mizutani, Y. Suzuoki, M. Hanai and M. Ieda, Jpn. J. Appl. Phys. 21, 1639 (1982).

${ }^{7}$ N. H. Ahmed and N. N. Srinivas, IEEE Trans. Dielectr. Electr. Insul. 4, 644 (1997).

${ }^{8} \mathrm{H}$. J. Wintle, in Proceedings of the 1998 3rd International Conference on Electric Charge in Solid Insulators, 29 June - 3 July 1998, Paris, France (Soc Francaise du Vide, Paris, France), pp. 49-58.

${ }^{9}$ G. Chen and Z. Xu, J. Appl. Phys. 106, 1237071 (2009).

${ }^{10}$ C. Tang, G. Chen, M. Fu, and R. Liao, IEEE Trans. Dielectr. Electr. Insul. 17(3), 775 (2010).

${ }^{11}$ G.-J. Zhang, K. Yang, M. Dong, W-B. Zhao, and Z. Yan, Appl. Surf. Sci. 254, 1450-(2007)

${ }^{12}$ G. C. Montanari, G. Mazzanti, F. Palmieri, A. Motori, G. Perego, and S. Serra, J. Phys. D: Appl. Phys. 34, 2902(2001).

${ }^{13}$ G. Chen, M. Fu, X. Z. Liu, and L. S. Zhong, J. Appl. Phys, 97, 083713 (2005).

${ }^{14}$ D. K. Das Gupta and M. K. Barbarez, J. Phys. D: Appl. Phys., 6, 867 (1973).

${ }^{15}$ K. C. Kao and W. Hwang, International Series in Science of the Solid State (Pergamon, Oxford, 1981), Vol. 14.

${ }^{16}$ C. K. Williams, J. Electron. Mater., 21(7), 711(1992).

${ }^{17}$ G. Chen, J. T. Sadipe, Y. Zhuang, C. Zhang, and G. C. Stevens, in Proceedings of the 9th International Conference on Properties and Applications of Dielectric Materials, Harbin, China, 19-23 July 2009, (IEEE, Piscataway, NJ, 2009), pp. 845-848.

${ }^{18}$ A. Tzimas, Ph.D. dissertation, University of Leicester, Leicester, 2008.

${ }^{19}$ A. Tzimas, S. M. Rowland, L. A. Dissado, M. Fu, and U. H. Nilsson, J. Phys. D: Appl. Phys. 43, 1(2010).

${ }^{20}$ M. Meunier and N. Quirke, J. Chem. Phys. 113(1), 369 (2000).

${ }^{21}$ D. Marsacq, P. Hourquebie, L. Olmedo, and H. Janah, in Proceedings of the 1995 Conference on Electrical Insulation and Dielectric Phenomena, Virginia Beach, VA, 22-25 October, 1995 (IEEE, Piscataway, NJ, 1995), pp. 672-675.

${ }^{22}$ G. Chen, J. Electrostat. 68(6), 487 (2010). 